\title{
A specialized post-anaesthetic care unit improves fast-track management in cardiac surgery: a prospective randomized trial
}

\author{
Stefan Probst ${ }^{1 * \dagger}$, Christof Cech ${ }^{1,2+}$, Dirk Haentschel ${ }^{3}$, Markus Scholz ${ }^{4}$ and Joerg Ender ${ }^{1}$
}

\begin{abstract}
Introduction: Fast-track treatment in cardiac surgery has become the global standard of care. We compared the efficacy and safety of a specialised post-anaesthetic care unit (PACU) to a conventional intensive care unit (ICU) in achieving defined fast-track end points in adult patients after elective cardiac surgery.

Methods: In a prospective, single-blinded, randomized study, 200 adult patients undergoing elective cardiac surgery (coronary artery bypass graft $(C A B G)$, valve surgery or combined CABG and valve surgery), were selected to receive their postoperative treatment either in the ICU $(n=100)$, or in the PACU $(n=100)$. Patients who, at the time of surgery, were in cardiogenic shock, required renal dialysis, or had an additive EuroSCORE of more than 10 were excluded from the study. The primary end points were: time to extubation (ET), and length of stay in the PACU or ICU (PACU/ICU LOS respectively). Secondary end points analysed were the incidences of: surgical re-exploration, development of haemothorax, new-onset cardiac arrhythmia, low cardiac output syndrome, need for cardiopulmonary resuscitation, stroke, acute renal failure, and death.
\end{abstract}

Results: Median time to extubation was $90[50 ; 140]$ min in the PACU vs. $478[305 ; 643]$ min in the ICU group ( $P$ $<0.001)$. Median length of stay in the PACU was $3.3[2.7 ; 4.0]$ hours vs. $17.9[10.3 ; 24.9]$ hours in the ICU $(P<0.001)$. Of the adverse events examined, only the incidence of new-onset cardiac arrhythmia (25 in PACU vs. 41 in ICU, P=0.02) was statistically different between groups.

Conclusions: Treatment in a specialised PACU rather than an ICU, after elective cardiac surgery leads to earlier extubation and quicker discharge to a step-down unit, without compromising patient safety.

Trial registration: ISRCTN71768341. Registered 11 March 2014.

\section{Introduction}

Anaesthesia for cardiac surgery has traditionally been provided with high-dose opioids and long-acting muscle relaxants, in the belief this technique was associated with optimal haemodynamic stability. The resulting prolonged postoperative ventilation and intensive care unit (ICU) length of stay (LOS) were considered acceptable compromises. Rising costs and the need for faster ICU turnover due to increased demand and reduced resources led to reducing the length of ICU stay after cardiac surgery $[1,2]$.

\footnotetext{
* Correspondence: stefan.probst@web.de

${ }^{\dagger}$ Equal contributors

'Department of Anaesthesiology and Intensive Care Medicine II, Leipzig Heart Centre, University of Leipzig, Struempellstrasse 39, Leipzig 04289, Germany

Full list of author information is available at the end of the article
}

Since the mid-1990s, intensified postoperative rehabilitation has established itself as the optimal approach to patient recovery. Fast-track treatment has become a popular and accepted standard because it allows for early extubation within six hours and consequently reduced LOS in the ICU and hospital [3-5]. A significant reduction in time to extubation (ET) without compromising patient's safety has been demonstrated in numerous studies [5-11]. Zhu et al. described in Cochrane Database Systematic Review a mean reduction of 5.99 hours (2.99 to 8.99 hours) due to implementation of a time-directed extubation protocol without increasing the risk of postoperative complications compared to standard care. Low-dose opioid anaesthesia will reduce mean ET by 7.40 hours (10.51 to 4.29 hours) compared to high-dose opioid anaesthesia [11]. 
Implementation of a dedicated fast-track protocol that allows not only for earlier extubation but also for earlier transfer from the ICU or post-anaesthetic care unit (PACU) to a step-down unit has been shown to be very effective in reducing ICU-LOS and the total length of hospital stay in retrospective studies [5,6,8]. Zhu et al. showed in a review that low-dose opioid anaesthesia was associated with 3.7 hours $(-6.98$ to -0.41$)$ lower ICU LOS. Time-directed extubation protocols had 5.15 hours $(-8.71$ to -1.59$)$ shorter length of stay in the ICU (0.4 to 8.7 hours) compared to conventional groups, as Zhu et al. described, although LOS in hospital was similar in both groups [11].

Utilised in combination, this approach has been associated with both significant cost savings, and also increased ICU bed capacity [12]. Most fast-track treatment protocols for cardiac surgery patients to date, however, have been implemented within the conventional ICU setting.

In general, it is possible to perform an extubation in the operating room (OR) with selected patient groups (OPCAB, MIDCAB and so on). This could make sense if no postoperative care unit is available or the fast-track concept is not continued at the ICU. There is still an ongoing discussion about the advantage of an early extubation in the OR. Straka et al. and Montes et al. were not able to show a reduced ICU LOS in cardiac surgery patients who get extubated in the OR $[13,14]$. Chamchad et al. found in a non-randomized observational study shorter ICU and hospital LOS. With an average ICU LOS of 27 hours, this study showed no additional benefit compared to early extubation in a PACU/ICU [15].

Nicholson et al. investigated in a randomized trial the effect of immediate extubation after coronary artery bypass graft (CABG) surgery compared to at least three hours ventilation before starting weaning on the pulmonary function. The study was performed in a PACU. They concluded that early extubation will not affect pulmonary function after extubation [16].

Our fast-track concept consists of direct postoperative treatment in a PACU with the primary goal of early extubation, followed by transfer to a step-down unit as soon as specific discharge criteria are met [6].

To the best of our knowledge, no prospective randomized study has been published which compares fast-track treatment in the ICU versus fast-track treatment in the PACU. The hypothesis of the study was that patients treated in the PACU would be extubated earlier, and be discharged to a step-down unit earlier than patients treated in the ICU. Accordingly, the objectives of our study were to compare ET and LOS in the PACU or ICU.

\section{Methods}

The study was approved by our local ethics committee (Ethics Committee, Medical Faculty, University of Leipzig,
Haertelstrasse 16-18, 04107 Leipzig, Reference number 097-2008, trial registration number ISRCTN71768341, http://www.controlled-trials.com/ISRCTN71768341/, registered 11 March 2014), and was conducted as a prospective, randomized, single-blinded, single-centre trial.

For each patient, written informed consent was obtained prior to any protocol-related activities. As part of this procedure, the principal investigator or designee explained orally and in writing the nature, duration, and purpose of the study in such a manner that the patient was aware of the potential risks, inconveniences, or adverse effects that may occur. The patient was informed that he/she was free to withdraw from the study at any time. The patient received all information that was required by local regulations and International Conference on Harmonisation (ICH) guidelines.

During the premedication visit the day before surgery, every patient scheduled to undergo CABG, valve surgery, or combined $C A B G$ and valve surgery was screened for inclusion in the study (Figure 1). Patients who were in cardiogenic shock, were dialysis dependent, or had an additive EuroSCORE of more than 10 were excluded.

The final decision for including or excluding the patient into the fast-track concept was taken by consensus decision between the attending anaesthesiologist and cardiac surgeon at the end of their surgery. Inclusion criteria were: haemodynamically stable (systolic blood pressure $>90 \mathrm{mmHg}$ and heart rate $<120 \mathrm{bpm}$; adrenaline or noradrenaline $<0.04 \mathrm{mcg} / \mathrm{kg} / \mathrm{min}$ ), normothermic $\left(>36^{\circ} \mathrm{C}\right.$ core body temperature), and no bleeding. Exclusion criteria followed risk factors identified by Constantinides et al. and Akhtar et al. [17,18]: impaired left ventricular function (ejection fraction below 35\%), cardiac assist devices pre- or postoperative and cardiopulmonary instability postoperative (high inotropic support, lactate $>5 \mathrm{mmol} / \mathrm{l}$, Horowitz index below 200) After the decision to include the patient into the study, the patient was randomized to either postoperative care in the PACU $(n=100)$ or ICU $(n=100)$. For that purpose an envelope was picked out of a box containing 200 sealed envelopes (100 for PACU, 100 for ICU admission) and removed from the box subsequently. A further intra-operative exclusion criterion was lack of an available bed in either the PACU or ICU. In such cases, the patient was not randomized, but was sent to the unit with an available bed, and excluded from the study and further analysis. The medical and nursing staff in the ICU and PACU had been informed about the design and the conduct of the study but were not informed as to which patients were enrolled in the study.

Data collection and analysis was performed by an independent person who was not part of the anaesthetic, surgical or ICU team, and who was not blinded to treatment allocation. 


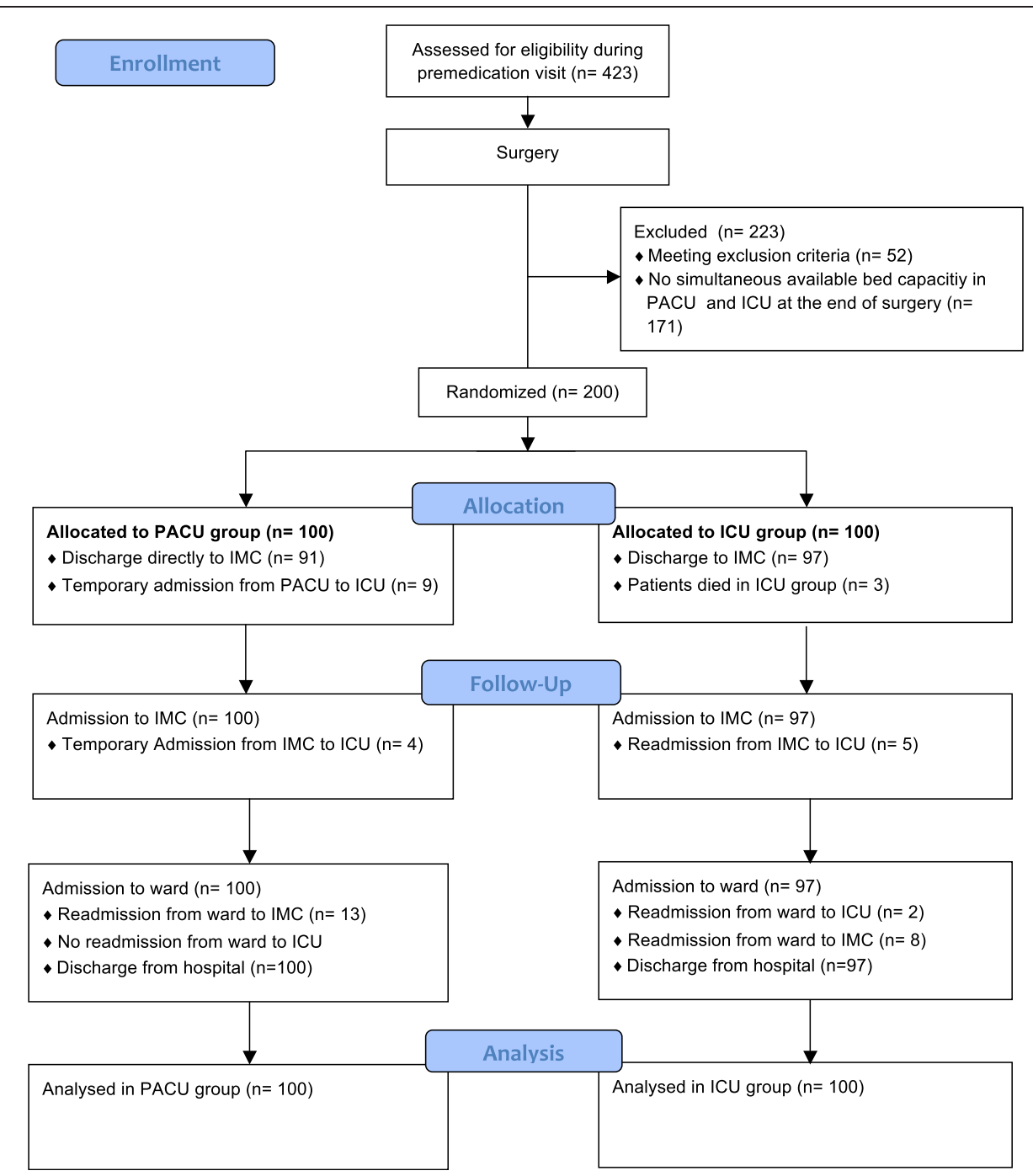

Figure 1 Study flowchart.

\section{Fast-track anaesthesia protocol}

Anaesthetic management consisted of oral premedication with clorazepate dipotassium (20 to $40 \mathrm{mg}$ ) the evening before and midazolam (3.75 to $7.5 \mathrm{mg}$ ) on the day of surgery. Anaesthesia was induced with fentanyl $(0.2 \mathrm{mg})$ and propofol $(1.5$ to $2 \mathrm{mg} / \mathrm{kg})$. A single dose of rocuronium $(0.6 \mathrm{mg} / \mathrm{kg})$ was used to facilitate intubation. Analgesia was maintained throughout the case with a continuous infusion of remifentanil $(0.2 \mathrm{mcg} / \mathrm{kg} / \mathrm{min})$, and for hypnosis during the pre- and post-cardiopulmonary bypass (CBP) period sevoflurane (0.8 to 1.1 minimum alveolar concentration (MAC)) was administered whereas during $\mathrm{CPB}$ a continuous propofol infusion $(3 \mathrm{mg} / \mathrm{kg} / \mathrm{h}$ ) was used. A recruitment manoeuvre was carried out prior to weaning from $\mathrm{CPB}$ in order to prevent atelectasis. An external convective warming system with an underbody blanket (Bairhugger $^{\mathrm{TM}}$, Arizant Healthcare; Eden Prairie, MN, USA) was used after weaning from $\mathrm{CPB}$ to ensure a core temperature of at least $36^{\circ} \mathrm{C}$ was maintained. For early postoperative analgesia, $1 \mathrm{~g}$ paracetamol was administered intravenously to each patient before skin closure. In difference to other studies, we did not include all patients or selected fast-track patients only preoperatively. All patients received the fast-track anaesthesia in the OR. We carefully selected fast-track patients at the end of surgery following the criteria identified as risk factors for fast-track failure $[1,17,18]$. The final decision to continue the fast-track protocol postoperatively was taken after the end of surgery. As our primary end point was postoperative ventilation time, we defined fast-track failure as postoperative ventilation of more than six hours. That was decided due the literature research where it ranged between three and nine hours $[19,20]$. 


\section{Treatment in PACU}

All patients were transferred to the PACU intubated, mechanically ventilated with a remifentanil infusion of 0.1 $\mathrm{mcg} / \mathrm{kg} / \mathrm{min}$. Administration of hypnotic agents was discontinued in the OR.

Postoperative analgesia consisted of an bolus of piritramide $(0.1 \mathrm{mg} / \mathrm{kg})$ on discontinuation of the remifentanil infusion, followed by bolus doses as required in 2 to $4 \mathrm{mg}$ aliquots, plus regular paracetamol ( 1 g every six hours) to achieve a pain score between 2 and 4 on an analogue pain scale from 0 to 10 . Patients were extubated when they were conscious and obeyed commands, had stable spontaneous ventilation with pressure support of 10 to 12 $\mathrm{cmH}_{2} \mathrm{O}$, positive end-expiratory pressure (PEEP) of 5 $\mathrm{cmH}_{2} \mathrm{O}$, fraction of inspired oxygen $\left(\mathrm{FiO}_{2}\right)$ of $\leq 0.4$, were haemodynamically stable, not bleeding $(\leq 100 \mathrm{ml} / \mathrm{h})$, and with no significant electrocardiographic abnormalities.

All patients received non-invasive bi-level positive airway pressure ventilation via a face mask for one hour (Elisee $350^{\mathrm{Tm}}$, Saime, Savigny-le-Temple, France), immediately after extubation. Initially non-invasive ventilation was commenced at a pressure support of 10 to $15 \mathrm{~cm}$ $\mathrm{H}_{2} \mathrm{O}$ and a PEEP of $5 \mathrm{cmH}_{2} \mathrm{O}$. The $\mathrm{FiO}_{2}$ was 0.4. During the period of non-invasive ventilation the pressure support was adapted to patients' needs.

Criteria for discharge to the intermediate care unit (IMC) were that patients must be awake, cooperative, haemodynamically stable (without inotropes) and have both acceptable respiratory pattern and blood gas analysis $\left(\mathrm{pO}_{2}>70 \mathrm{mmHg}, \mathrm{pCO}_{2}<50 \mathrm{mmHg}\right)$. Chest-X-ray and electrocardiogram were performed in all patients to exclude major pathology.

The physician-to-patient ratio and the nurse-to patientratio were 1:3. The PACU operated daily Monday to Friday from $10: 00$ to $18: 30$.

\section{Treatment in ICU}

All patients arrived in the ICU intubated, mechanically ventilated with a remifentanil infusion of $0.1 \mu \mathrm{g} / \mathrm{kg} / \mathrm{min}$. Administration of hypnotic agents was discontinued in the OR.

Postoperative analgesia consisted of a bolus of piritramide $(0.1 \mathrm{mg} / \mathrm{kg})$ on discontinuation of the remifentanil infusion, followed by bolus doses as required in 2 to $4 \mathrm{mg}$ aliquots, plus regular paracetamol (1 g every six hours). A pain scale was not used on a regular basis for assessing pain. The need for an analgesic medication was estimated by nurses. Extubation criteria were identical to those in the PACU. Non-invasive ventilation after extubation was not implemented routinely. Further treatment in the ICU was determined by the ICU physician according to German guidelines for intensive care treatment in cardiac surgery patients [21]. Criteria for suitability to transfer to IMC were identical to those in the PACU.
The physician-to patient-ratio was $1: 12$ and the nurseto-patient ratio was 1:2.

Substantial differences in PACU and ICU treatment are listed in Table 1.

\section{Outcomes}

Primary end points were ET and PACU/ICU LOS. Secondary outcome measures were hospital LOS, overall length of intensive care treatment (total ICT LOS), in-house mortality, low cardiac output, new-onset cardiac arrhythmia, respiratory failure requiring prolonged ventilation or reintubation and incidences of surgical re-exploration and renal failure.

PACU/ICU LOS is defined as LOS in the PACU or ICU from the end of surgery until discharge to another unit. Additionally, secondary PACU/ICU LOS includes readmissions from step-down units to ICU as well as additional ICU time after transfer from the PACU to ICU based on medical or organisational circumstances.

IMC LOS is defined as LOS in IMC until discharge to a general ward.

Primary ICT LOS is defined as overall length of intensive care treatment (ICT) in PACU/ICU + IMC.

Total ICT LOS is defined as overall length of ICT in the PACU + ICU + IMC including readmission to a unit of higher care grade than a general ward and transfer from the PACU to the ICU.

If patients were transferred from the PACU to the ICU in case of medical or organizational circumstances, they were still analysed as being in the PACU group, although additional ICU LOS was not calculated in PACU/ICU LOS but in secondary PACU/ICU LOS and total ICT LOS. PACU patients who had to stay past 18:30 were admitted to the ICU for further treatment and were evaluated as described above.

Low cardiac output was defined as central venous saturation of $<65 \%$ with a haematocrit of $>30 \%$. Cardiac arrhythmia included atrial fibrillation and atrioventricular block. Acute renal failure was defined as an increase in postoperative serum creatinine of at least three times the preoperative value, or a serum creatinine $>150 \mu \mathrm{mol} / \mathrm{l}$. Stroke was defined as a new transient or permanent motor or sensory deficit of central origin or unexplained coma.

\section{Statistical analysis}

Sample sizes were calculated on the basis of data from a previous retrospective study at our institution [6] using SPSS 16.0 (SPSS Inc, Chicago, IL, USA). Using this data, we estimated that ET in the ICU compared to ET time in the PACU would occur four hours later and that the standard deviation would be approximately $500 \mathrm{~min}$. We calculated that 93 patients per group would be required to demonstrate a significant reduction in ET with a power of $90 \%$ at significance level of $5 \%$. Accounting for 
Table 1 Substantial differences in PACU vs. ICU treatment

\begin{tabular}{|c|c|c|}
\hline & PACU group & ICU group \\
\hline Physician-to-patient ratio & $1: 3$ & $1: 12$ \\
\hline Nurse-to-patient ratio & $1: 3$ & $1: 2$ \\
\hline Physicians specialisation & All anaesthesiologists & Diverse specialisations (for example cardiac surgeon) \\
\hline Beds available & 3 bed unit & 21 bed unit \\
\hline Opening time & Limited opening time & Unlimited opening, 24 hours \\
\hline Patient population & $\begin{array}{l}\text { Only elective cardiac surgery patients after pre- and } \\
\text { postoperative evaluation of fast-track suitability that } \\
\text { PACU staff can focus on }\end{array}$ & $\begin{array}{l}\text { Mixed, as in the PACU but additionally patients in need of } \\
\text { physicians' attention due to multimorbidity and severe } \\
\text { diseases (for example non-fast-track patients) }\end{array}$ \\
\hline \multirow[t]{2}{*}{ Analgesia regime } & Strict regime as described in method section & \multirow[t]{2}{*}{ Performed more liberally according to nurses estimation } \\
\hline & Pain scale for pain assessment. & \\
\hline Timing of extubation & As soon as extubation criteria were met & $\begin{array}{l}\text { According to physicians' estimation under consideration } \\
\text { of overall situation on the ICU presupposed that extubation } \\
\text { criteria were met }\end{array}$ \\
\hline \multirow[t]{2}{*}{ Weaning protocol } & Performed by physician & Mainly nurse-driven \\
\hline & Good compliance to the protocol & $\begin{array}{l}\text { Compliance to the weaning protocol depended on the } \\
\text { actual workload }\end{array}$ \\
\hline Stop of analgosedation & $\begin{array}{l}\text { Remifentanil stopped at arrival (after paracetamol } \\
\text { and piritramid were administered }\end{array}$ & $\begin{array}{l}\text { Remifentanil stop according to disposition of the intensivist } \\
\text { under consideration of overall situation on the ICU }\end{array}$ \\
\hline Non-invasive ventilation & Performed routinely & Performed in only $4 \%$ of our population \\
\hline Discharge to step-down unit. & $\begin{array}{l}\text { Patient were discharged to step-down unit as soon } \\
\text { as they met discharge criteria }\end{array}$ & $\begin{array}{l}\text { Discharge to the step-down unit depended on need for } \\
\text { ICU beds }\end{array}$ \\
\hline
\end{tabular}

PACU, post-anaesthetic care unit; ICU, intensive care unit.

drop-outs and incomplete data, we aimed to recruit 100 patients per group.

Comparisons between the two independent groups (ICU vs. PACU) were performed using the Mann-Whitney $U$ test for continuous data, Mantel-Haenzsel test for categorically ordered data (for example New York Heart Association (NYHA) score) and Fisher's exact test for binary data (for example adverse events). A threshold of 0.05 was considered as significant. All analyses were performed using SPSS 18.0. Continuous parameters were described by median and interquartile range. Categorical data are described by class-wise allocation numbers. Binary data are described as number of events.

The primary end point of this study was time to extubation. We have not adjusted for multiple testing, so other comparisons are considered explorative.

\section{Results}

A total of 423 patients consented to participate in the study. All patients were scheduled for CABG, aortic valve replacement (AVR), mitral valve repair/replacement (MVR) or a combination of these procedures (Table 2). A total of 223 patients were excluded intraoperatively, due to a lack of capacity in either the ICU or PACU $(n=171)$, or because they were considered unsuitable for fast-track management at the end of their surgery, according to our criteria listed above $(\mathrm{n}=52)$. A total of 200 patients were therefore included in the study from May 2008 until July 2009, 100 in each group. There were significantly more female patients in the PACU group (36 vs. $22, P=0.04$ ) (Table 3 ). Patients randomized to the PACU group had significantly shorter surgery time $(170 \min [145 ; 195]$ vs. $190 \min [160 ; 230] ; P$ $<0.001)$ and anaesthesia time $(255 \mathrm{~min}[235 ; 285]$ vs. 270 min $[245 ; 313] ; P=0.02)$ than those in the ICU group (Table 3). Because of at most weak correlations with our primary outcomes, we decided not to adjust the analysis of primary outcomes for these imbalanced base-line variables (not shown). Cross-clamp (XCL) time (64 $\mathrm{min}$ $[51 ; 79]$ vs. $66 \min [51 ; 80] ; P=0.69)$ and total cardiopulmonary bypass time $(100 \mathrm{~min}[75 ; 127]$ vs. $99 \mathrm{~min}$ $[79 ; 122] ; P=0.91$ ) were not significantly different between groups. The number and type of operations performed in

Table 2 Operations performed

\begin{tabular}{llll}
\hline Type of surgery: & $\begin{array}{l}\text { PACU group } \\
(\mathbf{n}=\mathbf{1 0 0})\end{array}$ & $\begin{array}{l}\text { ICU group } \\
(\mathbf{n}=\mathbf{1 0 0})\end{array}$ & $\boldsymbol{P}$ value \\
\hline AVR (n) & 26 & 31 & 0.53 \\
MVR (n) & 33 & 27 & 0.44 \\
CABG on-pump (n) & 19 & 31 & 0.07 \\
CABG off-pump (n) & 22 & 11 & 0.06 \\
Combined procedures (\%) & 4 & 9 & 0.25
\end{tabular}

Combined procedures are valve replacement/repair + CABG or combined repair/ replacement of two valves. PACU, post-anaesthetic care unit; ICU, intensive care unit; AVR, aortic valve replacement; MVR, mitral valve replacement/repair; CABG, coronary artery bypass. 
Table 3 Demographic data (median and corresponding interquartile range)

\begin{tabular}{|c|c|c|c|}
\hline & PACU group $(n=100)$ & ICU group $(n=100)$ & $P$ value \\
\hline Patients (n) & 100 & 100 & \\
\hline Age (years) & $65[55 ; 72]$ & $66[57 ; 72]$ & 0.61 \\
\hline Gender (male/female) & $64 / 36$ & $78 / 22$ & 0.04 \\
\hline EuroSCORE (0-10 points) & $2[1 ; 4]$ & $2[1 ; 3]$ & 0.64 \\
\hline Ejection fraction in \% & $63[55 ; 66]$ & $60[51 ; 65]$ & 0.16 \\
\hline NYHA (NYHA 1-4) & $17 / 49 / 34 / 0$ & $16 / 56 / 26 / 2$ & 0.92 \\
\hline $\operatorname{COPD}(n)$ & 8 & 10 & 0.81 \\
\hline Neurological deficit (n) & 9 & 5 & 0.41 \\
\hline Peripheral vascular disease (n) & 13 & 8 & 0.36 \\
\hline Diabetes mellitus ( $n$ ) & 25 & 31 & 0.43 \\
\hline Renal insufficiency (n) & 6 & 14 & 0.06 \\
\hline Operative time (min) & $170[145 ; 195]$ & $190[160 ; 230]$ & $<0.001$ \\
\hline Anaesthesia time (min) & $255[235 ; 285]$ & $270[245 ; 313]$ & 0.02 \\
\hline CPB time (min) & $100[75 ; 127]$ & $99[79 ; 122]$ & 0.91 \\
\hline Cross-clamp time (min) & $64[51 ; 79]$ & $66[51 ; 80]$ & 0.69 \\
\hline
\end{tabular}

PACU, post anaesthetic care unit; ICU, intensive care unit; NYHA, New York Heart Association; COPD, chronic obstructive pulmonary disease; CPB, cardiopulmonary bypass.

both groups are listed in Table 2. There was no significant difference in type of surgery.

\section{Time to extubation}

The median extubation time in PACU group was significantly shorter than in the ICU group (90 min $[50 ; 140]$ vs. $478 \min$ [305; 643]; $P<0.001$; Figure 2, Table 4). In the PACU group $97 \%$ of the patients were extubated within six hours of admission whereas only $33 \%$ of the patients in the ICU group fulfilled the criteria for successful fast-tracking $(P<0.001)$ [5].

In the PACU group five patients required reintubation (three for resurgery, one because of a convulsion, and one for respiratory failure) compared to ten patients in ICU group (five for re-operation, four for respiratory failure, one for cardiopulmonary resuscitation). Additive ventilation time for reintubated patients was $930 \mathrm{~min}$ [330; 1315] in the PACU group vs. 990 min [646; 6375] in the ICU group (0.68).

Although the PACU had limited opening hours, time of arrival at the PACU seems not to have influence on ET.

\section{Length of stay in PACU, ICU, and hospital}

The median LOS for the patients in the PACU group was 3.3 hours [2.7; 4.0] compared to 17.9 hours [10.3; $24.9]$ for patients in the ICU group $(P<0.001$; Figure 3 , Table 4).

The median LOS in the IMC was 23.0 hours [19.9; $41.8]$ in the PACU group and 21.0 hours $[10.5 ; 28.8]$ in the ICU group $(P<0.004)$.

Overall length of ICT in the PACU + ICU + IMC including readmission to a unit of higher care grade than

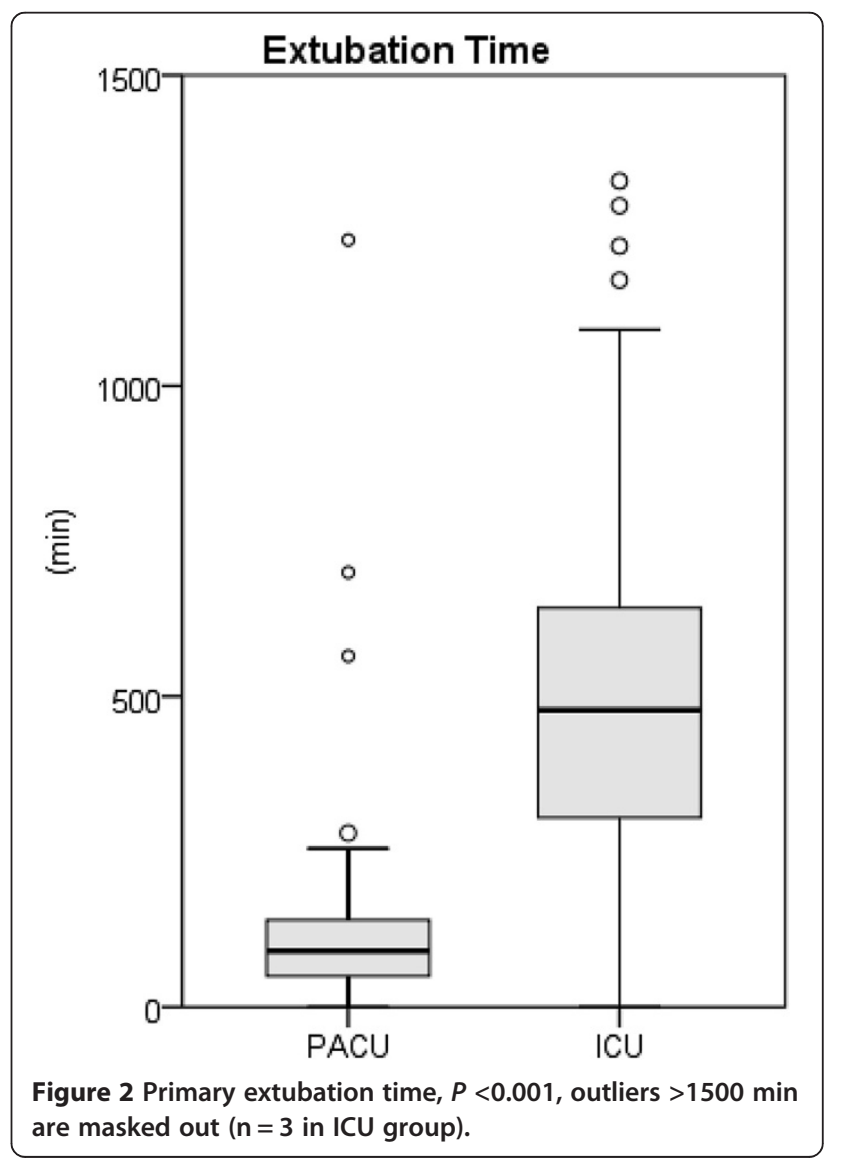


Table 4 Median extubation time and length of stay (LOS) and corresponding interquartile ranges

\begin{tabular}{|c|c|c|c|}
\hline & PACU group $(n=100)$ & ICU group $(n=100)$ & $P$ value \\
\hline Primary extubation time (min) & $90[50 ; 140]$ & $478[305 ; 643]$ & $<0.001$ \\
\hline Extubation within 6 h (n) & 97 & 33 & $<0.001$ \\
\hline Reintubation (n) & 5 & 10 & 0.28 \\
\hline Reintubation time (min) & $930[330 ; 1315]$ & $990[646 ; 6375]$ & 0.68 \\
\hline Total ventilation time (incl. reintubation) (min) & $105[70 ; 175]$ & $513[320 ; 705]$ & $<0.001$ \\
\hline PACU/ICU LOS (hours) & $3.3[2.7 ; 4.0]$ & $17.9[10.3 ; 24.9]$ & $<0.001$ \\
\hline Readmission to ICU (n) & 4 & 7 & 0.54 \\
\hline Secondary PACU/ICU LOS (hours) & $3.5[2.8 ; 5.1]$ & $17.9[10.3 ; 26]$ & $<0.001$ \\
\hline \multicolumn{4}{|c|}{ (incl. readmission from IMC to ICU and transfer from PACU to ICU) } \\
\hline Secondary PACU/ICU LOS <24 hours (n) & 95 & 71 & $<0.001$ \\
\hline Primary IMC LOS (hours) & $23.0[19.9 ; 41.8]$ & $21.0[10.5 ; 28.8]$ & 0.0035 \\
\hline Readmission IMC (n) & 13 & 8 & 0.09 \\
\hline Primary ICT LOS (hours) & $26.9[23.2 ; 46.0]$ & $41.1[24.8 ; 60.2]$ & 0.02 \\
\hline \multicolumn{4}{|l|}{ PACU + ICU + IMC excl. readmission } \\
\hline Total ICT LOS (hours) & $30.9[23.9 ; 59.9]$ & $43.9[24.9 ; 65.4]$ & 0.08 \\
\hline \multicolumn{4}{|c|}{ PACU + ICU + IMC incl. readmissions and transfer from PACU to ICU } \\
\hline Hospital LOS (d) & $9[8 ; 11]$ & $9[8 ; 12]$ & 0.42 \\
\hline
\end{tabular}

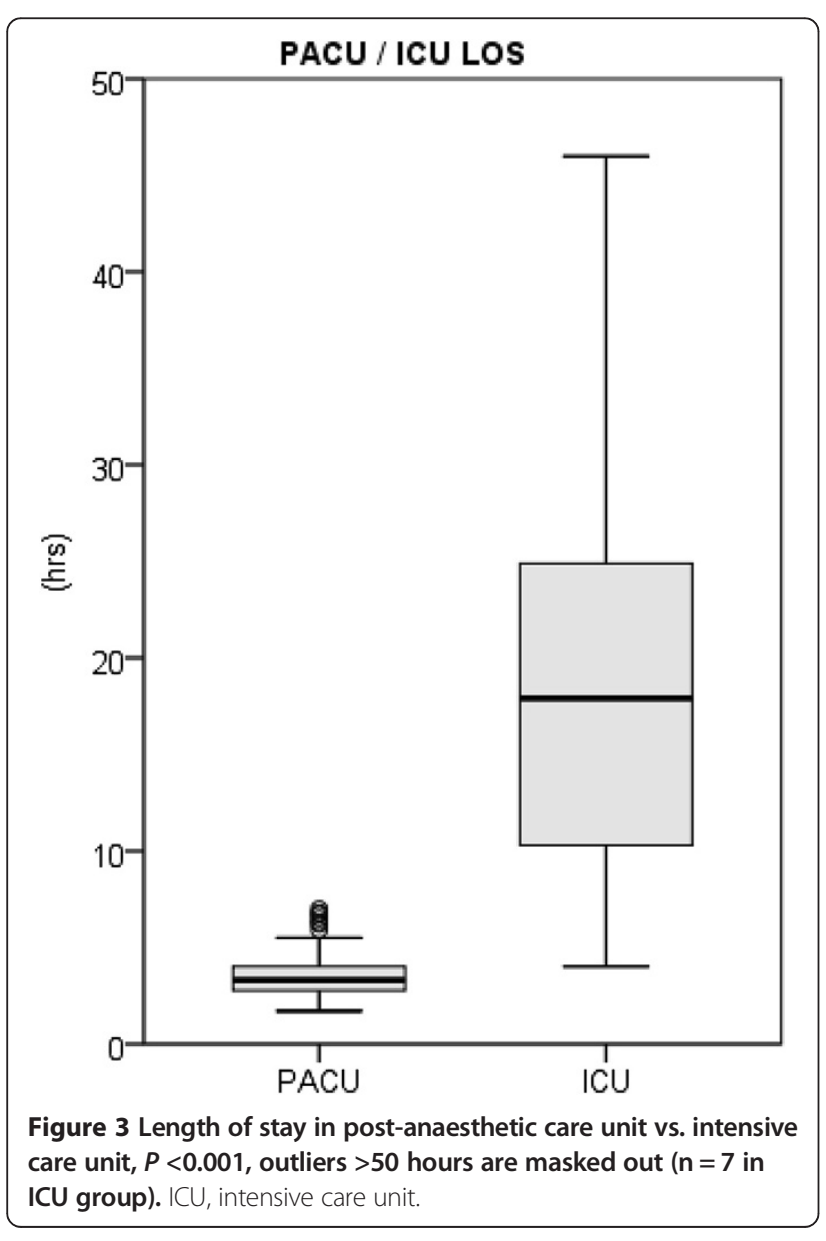

a general ward and transfer from the PACU to the ICU was 30.9 hours $[23.9$; 59.9] for patients in the PACU group compared to 43.9 hours $[24.9 ; 65.4]$ for patients in the ICU group $(P=0.08$; Figure 4 , Table 4$)$.

There was no significant difference in median hospital LOS for the PACU group $(9[8 ; 11])$ vs. the ICU group (9 $[8 ; 12]$ days).

Ninety-one of 100 patients in PACU group were discharged to intermediate care unit whereas nine patients had to be admitted from the PACU to the ICU (Figure 1). Three of these were extubated and haemodynamically stable, and were admitted to the ICU because of lack of available beds in IMC, two patients because of failure to extubate, two patients because of bleeding, and two patients because of cardiac arrhythmia. Four patients in the PACU group had to be admitted from IMC to the ICU (two because of re-thoracotomy, one because of haemodynamic instability, and one because of respiratory failure). A total of $87 \%$ of all patients in the PACU group did not require any treatment in the ICU.

Readmission from the general ward to IMC occurred in 13 patients of the PACU group, and was due to: cardiac arrhythmia $(n=4)$, pleural effusion $(n=5)$, pneumothorax $(n=2)$, resurgery $(n=1)$, and pain control $(n=1)$, no patient in the PACU group discharged to the ward required readmission to $\mathrm{ICU}$.

In the ICU group five patients required readmission from IMC to ICU, because of respiratory failure $(n=4)$ and cardiac arrest $(n=1)$. Two patients were readmitted 


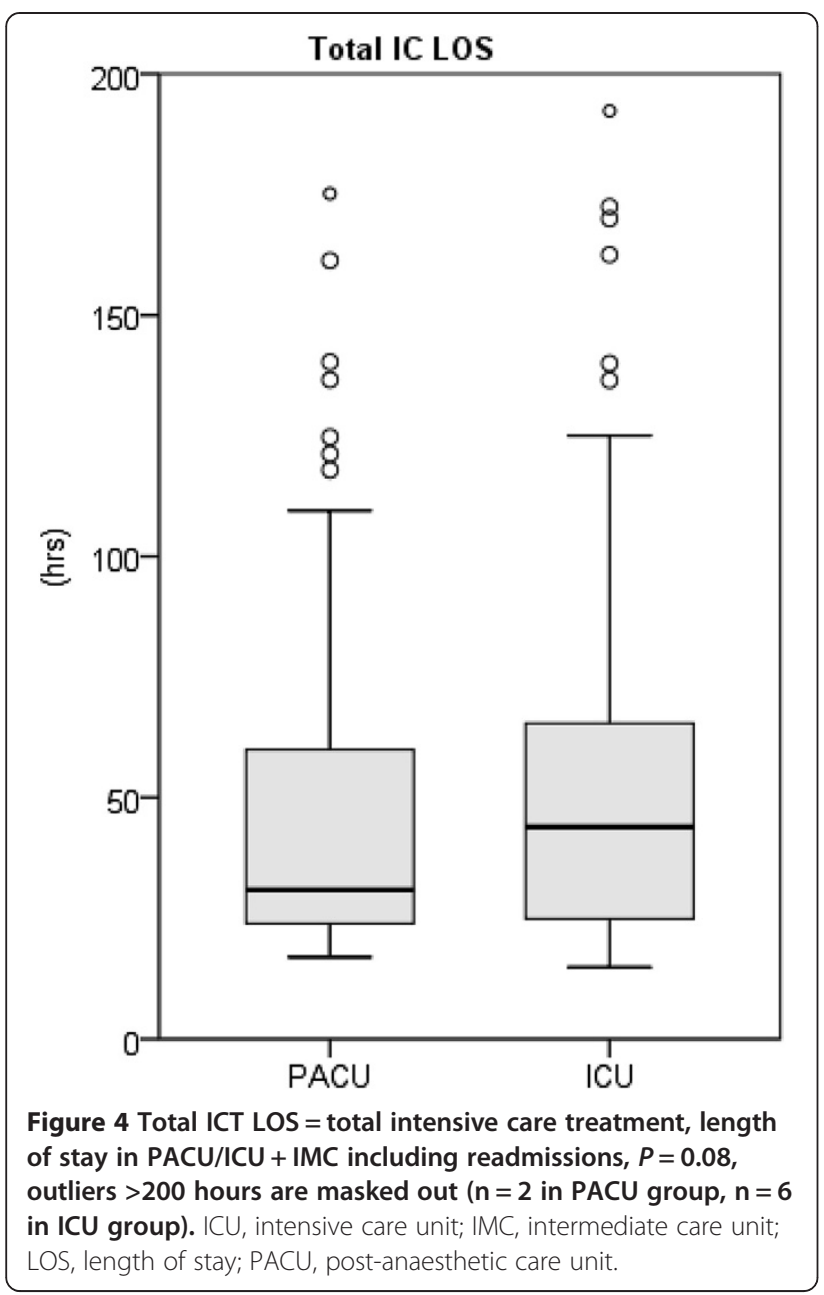

from the general ward to ICU because they required resurgery. Furthermore, in the ICU group eight patients had to be readmitted from the general ward to IMC because of cardiac arrhythmia $(n=5)$, neurological deficit $(\mathrm{n}=2)$ and pericardial effusion $(\mathrm{n}=1)$.

\section{Postoperative complications}

Postoperative complications for both groups are listed in Table 5. The occurrence of arrhythmias was significantly lower in the PACU group as compared to the ICU group (25 vs. $41, P=0.02$ ). There was no significant difference in the rate of pleural or pericardial effusions requiring intervention, renal insufficiency or cerebrovascular stroke.

The number of patients requiring resurgery (PACU $\mathrm{n}=5$ vs. ICU $\mathrm{n}=11, P=0.19)$ was lower in the PACU group (two for implantation of a pacemaker, two for drainage of a haemothorax, one for thrombectomy for deep vein thrombosis) compared to the ICU group (five for drainage of a haemothorax, two for revision of valve after valve replacement, two for implantation of a pacemaker, one thoracotomy for bleeding followed
Table 5 Postoperative complications

\begin{tabular}{|c|c|c|c|}
\hline & $\begin{array}{l}\text { PACU group } \\
(n=100)\end{array}$ & $\begin{array}{l}\text { ICU group } \\
(n=100)\end{array}$ & $P$ value \\
\hline Cardiac arrhythmia (n) & 25 & 41 & 0.02 \\
\hline Unstable sternum (n) & 1 & 1 & 1 \\
\hline $\begin{array}{l}\text { Pleural or pericardial } \\
\text { effusion }(n)\end{array}$ & 22 & 14 & 0.20 \\
\hline Renal failure (n) & 2 & 2 & 1 \\
\hline Reoperation (n) & 5 & 11 & 0.19 \\
\hline Stroke $(n)$ & 0 & 2 & 0.50 \\
\hline $\begin{array}{l}\text { Prolonged respiratory } \\
\text { insufficiency }>24 \text { hours (n) }\end{array}$ & 1 & 7 & 0.07 \\
\hline Low cardiac output (n) & 0 & 3 & 0.25 \\
\hline $\begin{array}{l}\text { Cardiopulmonary } \\
\text { resuscitation }(n)\end{array}$ & 0 & 5 & 0.06 \\
\hline Mortality (n) & 0 & 3 & 0.25 \\
\hline
\end{tabular}

PACU, post-anaesthetic care unit; ICU, intensive care unit.

by insertion of extracorporeal membrane oxygenation after resurgery, one for refixation of the sternum).

One patient from the PACU group required ventilation longer than 24 hours vs. seven patients in ICU group $(P=0.07)$.

Compared to ICU patients none of the PACU patients developed low cardiac output syndrome ( 3 vs. $0, P=0.25$ ), needed cardiopulmonary resuscitation (5 vs. $0, P=0.06$ ), or died ( 3 vs. $0, P=0.25$ ), although there was no statistically significant difference.

\section{Discussion}

In our study, we have shown that fast-track treatment of cardiac surgery patients in a dedicated PACU compared to fast-track treatment in the ICU significantly reduces ET (90 vs. $478 \mathrm{~min} ; P<0.001$ ) as well as time to transfer to a step-down unit (LOS PACU 3.3 hours compared to 17.9 hours LOS ICU). We were able to demonstrate a reduction of ventilation time and a significantly reduced utilisation of ICU capacity after cardiac surgery. Although we did not calculate the cost savings, Cheng et al. have clearly shown that early extubation results in reduced costs and better resource utilisation [4]. Hantschel et al. have also demonstrated that postoperative treatment in a PACU after cardiac surgery results in a $52 \%$ cost reduction compared to conventional ICU treatment [12]. Opening a PACU for 8.5 hours a day should lead to reduced personnel costs compared to a 24-hour ICU.

An ET of less than six hours after cardiac surgery is considered an important criterion for successful fasttracking after cardiac surgery $[4,5]$. In the PACU group $97 \%$ of the patients fulfilled this criterion but only $33 \%$ in the ICU group $(P<0.001)$. In a recent review, Zhu et al. showed that using a low-dose-opioid anaesthesia reduces ventilation times by 7.40 hours. Using a weaning protocol 
reduced ventilation times by 5.99 hours. In our study, we were able to reduce ventilation times by 6.46 hours, which is comparable to the reduction reported in other studies [11]. Our protocol used low-dose opioid anaesthesia with the short-acting opioid remifentanil. We defined a weaning protocol, which included early stop of anaesthesia, a protocol-driven postoperative pain management and non-invasive ventilation after extubation for at least 60 minutes.

Another fast-track criterion is reduced LOS in ICU, usually defined as less than 24 hours [5]. According to this criterion, successful fast-track-treatment was achieved in $95 \%$ of the PACU patients compared to $71 \%$ patients in the ICU group $(P<0.001)$. Zhu et al. reported in a review a reduction in ICU LOS for low-dose-opioid anaesthesia of 3.7 hours $(-6.98$ to -0.41$)$ and by using a weaning protocol of 5.15 hours ( -8.71 to -1.59$)$ compared to highdose-opioid anaesthesia [11]. In our study, we achieved a reduction in PACU/ICU LOS by 14.6 hours to 3.3 hours. This early discharge to a step-down unit allows using an ICU bed more than once a day. Gooch et al. developed a model of demand elasticity of ICU bed utilization [22]. The authors discussed that ICU beds created their own demand [23]. Under the model of demand elasticity the case mix of patients in the ICU changed depending on bed availability. If enough beds are available or no actual patient needs an ICU bed, it is more likely that patients in the ICU who are not as critically ill do not benefit from ICU stay [23]. By bypassing the ICU for fast-track patients, we possibly reduced this effect of demand elasticity and were able to show a reduction in ICU bed utilization. Still, if we included the readmission and direct transfers from the PACU to the ICU, we found a significant reduction for ICU LOS of 14.4 hours (secondary ICU LOS PACU vs. ICU 3.5 to 17.9 hours).

Published figures for fast-track failure rates range from $11 \%$ to $49 \%$ depending on the patient population $[17,18,24]$. In contrast to studies that included all patients undergoing cardiac surgery, our study population was preselected according to our existing fast-track protocol. We primarily excluded patients with a defined risk for fast-track failure during the premedication visit (patients who were scheduled for emergency surgery, were in cardiogenic shock, were dialysis dependent, or had an additive EuroSCORE of more than 10) $[1,17,25]$. Another explanation for the low fast-track failure rate of $5 \%$ for the PACU group is the fact that the final decision for inclusion of the patient to fast-track treatment was made at the end of the surgery. Wong et al. identified need for inotropic support and bleeding as risk factors for delayed extubation as well as delayed LOS in ICU [26]. In our study, 52 out of the 423 patients primarily included were excluded before randomisation because of hemodynamic instability or bleeding at the end of the operation. This underlines the hypothesis that not only careful preselection of potential fast-track patients during the premedication visit is important, but also that re-evaluation of patient suitability at the end of the operation can lead to a reduction of fast-track failure. The relatively high fast-track failure rate for the ICU group (67\% time to extubation $>6$ hours and 29\% PACU/ICU LOS $>24$ hours) may be attributable to several factors: first, the much better physician-to-patient ratio in the PACU (1:3 in the PACU vs. 1:12 in the ICU) allows the physician to effectively implement and manage an early goal-directed therapy. Since the study from Rivers et al. in septic patients we know that early hemodynamic stabilisation is beneficial for the patient and this is certainly also true for cardiac fast-track patients [27]. Several other studies have shown that an early goal-directed fluid management in postoperative cardiac surgery patients results in an improved hemodynamic stability and can reduce ventilation time and ICU LOS $[28,29]$. Second, due to the fact that one physician in the ICU cares for 12 patients the preselected fast-track patients will not get the same attention as the patient who really needs ICT. One to two severely compromised patients out of the 12 will result in the fact that weaning of the fast-track patient on ICU will be delayed. Kumar et al. have shown that the presence of an intensivist results in reduced ETs [30]. Third, the limited opening times for the PACU may positively motivate the involved staff to treat the patients optimally including early extubation and hemodynamic and respiratory stabilisation so that the patient can be transferred to the IMC for further treatment.

Also, the more focused adherence to the fast-track and enhanced-recovery principles including specifications for medication, postoperative pain control and discharge criteria favours the PACU compared to the ICU. van Mastrigt et al. showed in a meta-analysis that a defined weaning-and-extubation protocol is an important key to reduced intensive care LOS [10]. Although this protocol was the same for the PACU and the ICU, the more disciplined execution of the fast-track protocol and application of non-invasive ventilation in our PACU might be another important factor for success of early extubation. In a prospective randomized study, Zarbock et al. demonstrated a significant reduction in reintubation and readmission to ICU/IMC in cardiac surgery patients using continuous positive airway pressure therapy [31].

We found a lower incidence of reintubation in the PACU with $2.5 \%$ (five) vs. $5 \%$ in the ICU (ten) patients and a lower readmission rate of the PACU (four) vs. the ICU (seven) patients from step-down unit (IMC) to the ICU without reaching significance. Zhu et al. reported a risk of reintubation in the fast-track group of $1.4 \%$ and in the conventional group of $1.7 \%$, [11], which is lower 
as in our study. However, this study is underpowered to allow any conclusion to the reintubation rate compared to other studies.

The incidences of low cardiac output syndrome, prolonged respiratory insufficiency, cardiac arrest, and death tended to be lower in the PACU group without reaching statistical significance. Because these complications were not primary end points, our study was underpowered for demonstrating significant differences between groups. The incidence of renal failure, stroke, resurgery, and mortality was similar for the PACU and the ICU group. Our study does not allow any conclusion about the safety of our fast-track concept. However, a significantly lower incidence of common postoperative complications for fast-track patients was demonstrated in a prospective study of 1,488 patients by Gooi et al. [3]. Svircevic et al. could not find any evidence for increased risk of adverse outcomes in 7,989 patients undergoing fast-track cardiac surgery [5]. In a recent review, Zhu et al. came to the conclusion that fast-track interventions have similar risks of mortality and major postoperative complications to conventional (not fast-track) care, and therefore appear to be safe in patients considered to be at low to moderate risk [11].

In contrast to other studies on fast-track in cardiac surgery, which included only patients undergoing coronary artery bypass surgery, our patient population was mixed regarding type of operations [4,10,19,32]. More than half of our patient population underwent valve surgery, some of them in combination with CABG. Overall, in our patient population of $n=200$ patients only $41.5 \%$ were CABG patients (41 vs. 42 ). A total of $6.5 \%$ of all patients (four vs. nine) underwent combined procedures (for example aortic and mitral valve surgery or valve surgery and $C A B G)$. We have also shown that fast-track treatment utilising a dedicated PACU can be successfully implemented for different types of cardiac operations.

\section{Limitations of the study}

Our demographic data show that there is a significant difference in gender (more female patients in the PACU group). In several studies, female gender was found to be a risk factor for delayed postoperative extubation and prolonged ICU length of stay $[1,26]$. This might have favoured the ICU group. Anaesthesia and surgery time in the ICU group was significantly longer, but there was no difference in XCL and cardiopulmonary bypass time, which were (amongst others) identified as risk factors for delayed postoperative extubation ( $>6$ hours) and prolonged ICU LOS ( $>24$ hours) $[1,26]$. Regarding anaesthesia and surgery time, we observed only weak correlations with our outcome variables in both PACU and ICU groups. Hence, it is unlikely that this imbalance in baseline characteristics affects the main conclusion of our study.
Regarding the adverse events, the study was not adequately powered to identify significant differences between the groups.

\section{Conclusions}

Our study showed that our fast-track treatment in a dedicated PACU leads to a high rate of success (95\%) compared to the ICU (33\%). We attribute this difference to better physician-to-patient ratio, allowing for more focused, early postoperative management, and better adherence to an established fast-track protocol. Delaying the decision about patient suitability for fast-track treatment until the end of surgery may also contribute to reducing the incidence of fast-track failures. Running a PACU separated from the ICU in a different part of the hospital, an excellent physician-patient ratio and strong adherence to the fast-track protocol is from our point of view one of the success factors for our study.

\section{Key messages}

- ET for cardiac surgery patients in a fast-track protocol is significantly shorter in a dedicated PACU than in ICU

- PACU-LOS is significantly shorter than ICU-LOS

\section{Abbreviations}

AVR: aortic valve replacement; CABG: coronary artery bypass graft; CBP: cardiopulmonary bypass; COPD: chronic obstructive pulmonary disease; ET: time to extubation; $\mathrm{FiO}_{2}$ : fraction of inspired oxygen; ICT: intensive care treatment; ICU: intensive care unit; IMC: intermediate care unit; LOS: length of stay; MAC: minimum alveolar concentration; MVR: mitral valve replacement/repair; NYHA: New York Heart Association; OR: operating room; PACU: post-anaesthetic care unit; PEEP: positive end-expiratory pressure; XCL: cross-clamp.

\section{Competing interests}

The authors declare that they have no competing interests.

\section{Authors' contributions}

SP contributed to conception and design of the study, interpretation of data, manuscript writing and final approval of the manuscript. CC contributed to conception and design of the study, acquisition of patients, data collection, statistical analysis and interpretation, manuscript writing and final approval of the manuscript. DH contributed to conception and design of the study, acquisition of patients, data collection, manuscript writing and final approval of the manuscript. MS contributed to statistical analysis, interpretation of data, critical revision, manuscript writing and final approval of the manuscript. JE contributed to conception and design of the study, coordination, archiving the study files, manuscript writing and final approval of the manuscript. All authors read and approved the final manuscript.

Authors' information

Stefan Probst and Christof Cech: co-first authors.

\section{Acknowledgment}

The study was funded entirely by the host institution. Approved by: Ethics Committee, Medical Faculty, University of Leipzig, Haertelstrasse 16-18, 04107 Leipzig, Reference number 097-2008. 


\section{Author details}

${ }^{1}$ Department of Anaesthesiology and Intensive Care Medicine II, Leipzig Heart Centre, University of Leipzig, Struempellstrasse 39, Leipzig 04289, Germany. ${ }^{2}$ Department of Anaesthesiology and Intensive Care Medicine, University of Leipzig, Medical Faculty, Liebigstrasse 20, Leipzig 04103, Germany. 'Department of Anaesthesiology and Intensive Care Medicine, Heart Centre Coswig, Lerchenfeld 1, Coswig 06869, Germany. ${ }^{4}$ Institute of Medical Informatics, Statistics, and Epidemiology, University of Leipzig, Härtelstraße 16-18, Leipzig 04107, Germany.

Received: 18 February 2014 Accepted: 22 July 2014 Published: 15 August 2014

\section{References}

1. Lassnigg A, Hiesmayr MJ, Bauer P, Haisjackl M: Effect of centrepatient- and procedure-related factors on intensive care resource utilisation after cardiac surgery. Intensive Care Med 2002, 28:1453-1461.

2. Silbert BS, Myles PS: Is fast-track cardiac anesthesia now the global standard of care? Anesth Analg 2009, 108:689-691.

3. Gooi J, Marasco S, Rowland M, Esmore D, Negri J, Pick A: Fast-track cardiac surgery: application in an Australian setting. Asian Cardiovasc Thorac Ann 2007, 15:139-143.

4. Cheng DC, Karski J, Peniston C, Raveendran G, Asokumar B, Carroll J, David T, Sandler A: Early tracheal extubation after coronary artery bypass graft surgery reduces costs and improves resource use. A prospective, randomized, controlled trial. Anesthesiology 1996, 85:1300-1310.

5. Svircevic V, Nierich AP, Moons KG, Brandon Bravo Bruinsma GJ, Kalkman CJ, Van DD: Fast-track anesthesia and cardiac surgery: a retrospective cohort study of 7989 patients. Anesth Analg 2009, 108:727-733.

6. Ender J, Borger MA, Scholz M, Funkat AK, Anwar N, Sommer M, Mohr FW, Fassl J: Cardiac surgery fast-track treatment in a postanesthetic care unit: six-month results of the Leipzig fast-track concept. Anesthesiology 2008, 109:61-66.

7. Moon MC, Abdoh A, Hamilton GA, Lindsay WG, Duke PC, Pascoe EA, Del Rizzo DF: Safety and efficacy of fast track in patients undergoing coronary artery bypass surgery. J Card Surg 2001, 16:319-326.

8. Flynn M, Reddy S, Shepherd W, Holmes C, Armstrong D, Lunn C, Khan K, Kendall S: Fast-tracking revisited: routine cardiac surgical patients need minimal intensive care. Eur J Cardiothorac Surg 2004, 25:116-122.

9. Cheng DC, Karski J, Peniston C, Asokumar B, Raveendran G, Carroll J, Nierenberg H, Roger S, Mickle D, Tong J, Zelovitsky J, David T, Sandler A: Morbidity outcome in early versus conventional tracheal extubation after coronary artery bypass grafting: a prospective randomized controlled trial. J Thorac Cardiovasc Surg 1996, 112:755-764.

10. van Mastrigt GA, Maessen JG, Heijmans J, Severens JL, Prins MH: Does fast-track treatment lead to a decrease of intensive care unit and hospital length of stay in coronary artery bypass patients? A meta-regression of randomized clinical trials. Crit Care Med 2006, 34:1624-1634.

11. Zhu F, Lee A, Chee YE: Fast-track cardiac care for adult cardiac surgical patients. Cochrane Database Syst Rev 2012, 10, CD003587.

12. Hantschel D, Fassl J, Scholz M, Sommer M, Funkat AK, Wittmann M, Ender J: [Leipzig fast-track protocol for cardio-anesthesia. Effective, safe and economical]. Anaesthesist 2009, 58:379-386.

13. Straka Z, Brucek P, Vanek T, Votava J, Widimsky P: Routine immediate extubation for off-pump coronary artery bypass grafting without thoracic epidural analgesia. Ann Thorac Surg 2002, 74:1544-1547.

14. Montes FR, Sanchez SI, Giraldo JC, Rincon JD, Rincon IE, Vanegas MV, Charris $\mathrm{H}$ : The lack of benefit of tracheal extubation in the operating room after coronary artery bypass surgery. Anesth Analg 2000, 91:776-780.

15. Chamchad D, Horrow JC, Nachamchik L, Sutter FP, Samuels LE, Trace CL, Ferdinand F, Goldman SM: The impact of immediate extubation in the operating room after cardiac surgery on intensive care and hospital lengths of stay. J Cardiothorac VasC Anesth 2010, 24:780-784.

16. Nicholson DJ, Kowalski SE, Hamilton GA, Meyers MP, Serrette C, Duke PC: Postoperative pulmonary function in coronary artery bypass graft surgery patients undergoing early tracheal extubation: a comparison between short-term mechanical ventilation and early extubation. J Cardiothorac Vasc Anesth 2002, 16:27-31.
17. Constantinides VA, Tekkis PP, Fazil A, Kaur K, Leonard R, Platt M, Casula R, Stanbridge R, Darzi A, Athanasiou T: Fast-track failure after cardiac surgery: development of a prediction model. Crit Care Med 2006, 34:2875-2882

18. Akhtar Ml, Hamid M: Success and failure of fast track extubation in cardiac surgery patients of tertiary care hospital: one year audit. J Pak Med Assoc 2009, 59:154-156.

19. Cheng DC, Newman MF, Duke P, Wong DT, Finegan B, Howie M, Fitch J, Bowdle TA, Hogue C, Hillel Z, Pierce E, Bukenya D: The efficacy and resource utilization of remifentanil and fentanyl in fast-track coronary artery bypass graft surgery: a prospective randomized, double-blinded controlled, multi-center trial. Anesth Analg 2001, 92:1094-1102.

20. Lison S, Schill M, Conzen P: Fast-track cardiac anesthesia: efficacy and safety of remifentanil versus sufentanil. J Cardiothorac Vasc Anesth 2007, 21:35-40.

21. Carl M, Alms A, Braun J, Dongas A, Erb J, Goetz A, Goepfert M, Gogarten W Grosse J, Heller AR, Heringlake M, Kastrup M, Kroener A, Loer SA, Marggraf G, Markewitz A, Reuter D, Schmitt DV, Schirmer U, Wiesenack C, Zwissler B, Spies C: S3 guidelines for intensive care in cardiac surgery patients: hemodynamic monitoring and cardiocirculatory system. Ger Med Sci 2010, 13:1-25

22. Gooch RA, Kahn JM: ICU bed supply, utilization, and health care spending: an example of demand elasticity. JAMA 2014, 311:567-568.

23. Wunsch $\mathrm{H}$, Angus DC, Harrison DA, Linde-Zwirble WT, Rowan KM: Comparison of medical admissions to intensive care units in the United States and United Kingdom. Am J Respir Crit Care Med 2011, 183:1666-1673.

24. Lee TW, Grocott HP, Schwinn D, Jacobsohn E: High spinal anesthesia for cardiac surgery: effects on beta-adrenergic receptor function, stress response, and hemodynamics. Anesthesiology 2003, 98:499-510.

25. Toraman F, Senay S, Gullu U, Karabulut H, Alhan C: Readmission to the intensive care unit after fast-track cardiac surgery: an analysis of risk factors and outcome according to the type of operation. Heart Surg Forum 2010, 13:E212-E217.

26. Wong DT, Cheng DC, Kustra R, Tibshirani R, Karski J, Carroll-Munro J, Sandler A: Risk factors of delayed extubation, prolonged length of stay in the intensive care unit, and mortality in patients undergoing coronary artery bypass graft with fast-track cardiac anesthesia: a new cardiac risk score. Anesthesiology 1999, 91:936-944.

27. Rivers EP, Kruse JA, Jacobsen G, Shah K, Loomba M, Otero R, Childs EW: The influence of early hemodynamic optimization on biomarker patterns of severe sepsis and septic shock. Crit Care Med 2007, 35:2016-2024.

28. Kapoor PM, Kakani M, Chowdhury U, Choudhury M, Lakshmy, Kiran U: Early goal-directed therapy in moderate to high-risk cardiac surgery patients. Ann Card Anaesth 2008, 11:27-34

29. McKendry M, McGloin H, Saberi D, Caudwell L, Brady AR, Singer M: Randomised controlled trial assessing the impact of a nurse delivered, flow monitored protocol for optimisation of circulatory status after cardiac surgery. BMJ 2004, 329:258.

30. Kumar K, Zarychanski R, Bell DD, Manji R, Zivot J, Menkis AH, Arora RC: Impact of 24-hour in-house intensivists on a dedicated cardiac surgery intensive care unit. Ann Thorac Surg 2009, 88:1153-1161.

31. Zarbock A, Mueller E, Netzer S, Gabriel A, Feindt P, Kindgen-Milles D: Prophylactic nasal continuous positive airway pressure following cardiac surgery protects from postoperative pulmonary complications: a prospective, randomized, controlled trial in 500 patients. Chest 2009, 135:1252-1259.

32. Tempe DK, Gandhi A, Virmani S: Resource utilization in on- and off-pump coronary artery surgery: factors influencing postoperative length of stay-an experience of 1,746 consecutive patients undergoing fast-track cardiac anesthesia. J Cardiothorac Vasc Anesth 2006, 20:128-129.

doi:10.1186/s13054-014-0468-2

Cite this article as: Probst et al:: A specialized post-anaesthetic care unit improves fast-track management in cardiac surgery: a prospective randomized trial. Critical Care 2014 18:468. 